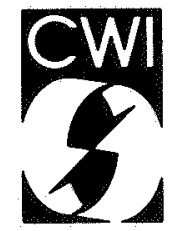

Centrum voor Wiskunde en Informatica Centre for Mathematics and Computer Science

V.V. Slavova

Berry-Esseen bound for Student's statistic 
The Centre for Mathematics and Computer Science is a research institute of the Stichting Mathematisch Centrum, which was founded on February 11, 1946, as a nonprofit institution aiming at the promotion of mathematics, computer science, and their applications. It is sponsored by the Dutch Government through the Netherlands Organization for the Advancement of Pure Research (Z.W.O.).

Copyrighi ic Stichting Mathematisch Centrum, Amsterdam 


\title{
Berry-Esseen Bound for Student's Statistic
}

\author{
V.V. Slavova \\ All Soviet Institute of Medical and Medical Technical Information of \\ Ministry of Health, Moscow
}

\begin{abstract}
The Berry-Esseen bound for the distribution of Student's $t$-statistic is obtained under the sole condition that the underlying distribution has a finite 3rd moment.

1980 Mathematics Subject Classification: 60F05, 62E20.

Key Words \& Phrases: Berry-Esseen bound, Student $t$-statistic.

Note: This paper appeared in Stability Problems for Stochastic Models, V.V. Kalashnikov and V.M. Zolotarev eds. Lecture Notes in Mathematics, No. 1155, Springer-Verlag 1985. p.p. 355-390 with title 'On the BerryEsseen bound for Student's statistic'. The permission given by Springer-Verlag to reproduce the paper as a CWI report is gratefully acknowledged.
\end{abstract}

\section{0 . INTRODUCTION}

We establish the Berry-Esseen bound for the distribution of Student's $t$-statistic under the sole assumption that the underlying distribution has a finite 3rd moment (see $(1.1-3)$ below).

There are a number of results of Berry-Esseen type for various classes of statistics, e.g., for various kinds of estimators, for linear combinations of order statistics, for $U$-statistics etc. The case of Student's $t$ was considered in [1] - [3]. In [1], it was assumed that the observations, $X_{i}$, have a finite moment of order $r=6$. This assumption makes it possible to apply the asymptotic theory for sums of i.i.d. random variables in a rather straightforward way because $t$ involves the sums of $X^{\prime} s$ and $X^{2}$ 's, these summands having then a finite 3rd moment.

In a recent paper [3] the Berry-Esseen bound for Student's $t$ was obtained under the condition that $r>\frac{10}{3}$. (The authors of [3] require that $r>4$ but they point out that this is only needed to reduce $t$ to a $U$-statistic and it appears that this reduction is valid for $r>\frac{10}{3}$ as well.)

A closely related result is that of [5] from which the Edgeworth expansion for the distribution of $t$ can be obtained. In particular, the one-term Edgeworth expansion is valid under the present condition that $r=3$ and an additional assumption that the characteristic function of the joint distribution of $\left(X_{1}, X_{1}^{2}\right)$ satisfies Cramérs condition $(C)$.

In the present paper no conditions of the latter type are imposed. This is the main cause for differences between our proof and that of [5]. In the proof to follow we mostly omit the parts which can be carried through by specializing the corresponding parts of the proof in [5] and present in full detail those which are essentially different. 
1. The MaIN RESUlt.

Let $X_{1}, \ldots, X_{n}$ be i.i.d. random variables such that

$$
E X_{1}=0, \quad E X_{1}^{2}=1, \quad \nu_{3}=E\left|X_{1}\right|^{3}<\infty .
$$

Consider Student's statistic

$$
t=\frac{n^{\frac{1}{2}} \bar{X}}{s}
$$

where

$$
\bar{X}=n^{-1} \Sigma X_{i}, \quad s^{2}=(n-1)^{-1} \Sigma\left(X_{i}-\bar{X}\right)^{2} .
$$

(Unless otherwise stated, the subscript $i$ runs over $\{1, \ldots, n\}$.)

THEOREM. Under the condition (1.1) there exists a constant $C=C\left(\nu_{3}\right)$ such that, for all $n \geqslant 2$,

$$
\sup _{x}|P\{t<x\}-\Phi(x)| \leqslant C n^{-\frac{1}{2}},
$$

where $\Phi$ denotes the standard normal distribution function.

The results of this type were obtained in [1], [2] and [3] under more restrictive conditions.

Proor. Let

$$
Y_{i}=X_{i}^{2}-1, \quad S_{n}=n^{-\frac{1}{2}} \sum X_{i}, \quad T_{n}=n^{-\frac{1}{2}} \sum Y_{i}
$$

Then $t$ can be rewritten as

$$
t=\left(\frac{n-1}{n}\right)^{\frac{1}{2}} \frac{S_{n}}{\left(1+\frac{1}{\sqrt{n}} T_{n}-\frac{1}{n} S_{n}^{2}\right)^{\frac{1}{2}}} .
$$

The arguments used in the proof of the corollary (4.1) [4] show that (1.3) will follow if we prove that for any $a \in R$ there exists $C_{1}=C_{1}\left(a, \nu_{3}\right)$ such that

$$
\sup _{x}\left|P\left\{S_{n}-\frac{1}{2 \sqrt{n}} S_{n} T_{n}+\frac{a}{n} S_{n} T_{n}^{2}<x\right\}-\Phi(x)\right| \leqslant \frac{C_{1}}{\sqrt{n}} .
$$

Note that it is sufficient to prove (1.3) or (1.6) for all large enough $n$ since for, say, $n \leqslant n_{0},(1.3)$ or (1.6) trivially hold true with $C$ (or $\left.C_{1}\right)=\sqrt{n_{0}}$.

The proof of (1.6) follows the lines of that of Theorem of [5]. The differences are due to obtaining inequalities rather than o-estimates for the remainders. Moreover we don't impose any "smoothness" conditions like Cramér's condition $(C)$ in [5], which requires a different manner of smoothing the sums, $S_{n}, T_{n}$, entering into (1.6).

Let $\xi_{1}, \ldots, \xi_{n}, \eta$ be mutually independent and independent from $\left\{X_{i}\right\}$ r.v.'s, $\xi_{i}$ being identically distributed as $N\left(o, \sigma^{2}\right)$ (normal with zero mean and variance $\sigma^{2}$ ) and $\eta$ having the characteristic function $\theta\left(\frac{s}{A}\right)$, where $\theta(s)$ is an even twice differentiable characteristic function vanishing outside $[-1,1]$. The parameters $\sigma^{2}=\sigma^{2}\left(\nu_{3}\right)$ and $A=A\left(\nu_{3}\right)$ will be specified in the paragraph following (1.16). We introduce the following truncated and smoothed variables

$$
\begin{aligned}
& \tilde{X}_{i}=X_{i} 1_{\left\{\left|X_{i}\right| \leqslant \sqrt{n}\right\}}-E X_{i} 1_{\left\{\left|X_{i}\right| \leqslant \sqrt{n}\right\}}, \\
& \tilde{Y}_{i}=Y_{i} 1_{\left\{\left|Y_{i}\right| \leqslant n-1\right\}}-E Y_{i} 1_{\left\{\left|Y_{i}\right| \leqslant n-1\right\}}, \quad Y_{i}^{*}=\tilde{Y}_{i}+\xi_{i}, \\
& \tilde{S}_{n}=n^{-\frac{1}{2}} \sum \tilde{X}_{i}, \quad S_{n}^{*}=\tilde{S}_{n}+\frac{\eta}{\sqrt{n}}, \quad T_{n}^{*}=n^{-\frac{1}{2}} \sum Y_{i}^{*} .
\end{aligned}
$$


Denote by $p_{n}^{*}(x, u)$ and $r_{n}^{*}(x)$ the density functions of $\left(S_{n}^{*}, T_{n}^{*}\right)$ and $S_{n}^{*}$ and let

$$
\pi_{n}(x, u)=\sup _{y:|y-x| \leqslant 1}\left|D_{y} p_{n}^{*}(y, u)\right| \text {. }
$$

Here and in what follows we write $D_{y}$ and $D_{y}^{k}$ for $\frac{\partial}{\partial y}$ and $\frac{\partial^{k}}{\partial y^{k}}, k=2,3, \ldots$ Let, further,

$$
B_{n}=\{x:|x| \leqslant 2 \ln n-1\}, \quad E_{x}=\left\{u:|u| \leqslant a_{n}(x)\right\}
$$

with

$$
a_{n}(x)=\frac{\sqrt{n}}{(1+|x|)^{2}}-1
$$

In what follows we denote by $C$ constants (not necessarily the same in different places) depending on $\nu_{3}$ and possibly on some other parameters. The use of this symbol will mean an assertion that such a constant exists.

By the arguments similar to those in Sections 3,4,5 and 10 of [5] the proof of (1.6) is reduced to the proof of the following relations:

There exist $A_{0}=A_{0}\left(\nu_{3}\right)$ and $N_{0}=N_{0}\left(\nu_{3}, A, \sigma\right)$ such that, for any $0<A \leqslant A_{0}, \sigma>0, n \geqslant N_{0}$

$$
\begin{aligned}
& \sup _{x \in B_{n}} E\left\{S_{n}^{*} T_{n}^{*} \mid S_{n}^{*}=x\right\} r_{n}^{*}(x) \leqslant C(A, \sigma) \\
& \sup _{x \in B_{n}} E\left\{S_{n}^{*} T_{n}^{* 2} \mid S_{n}^{*}=x\right\} r_{n}^{*}(x) \leqslant \sqrt{n} C(A, \sigma) .
\end{aligned}
$$

There exist $A_{1}=A_{1}\left(\nu_{3}, \sigma\right), \sigma_{1}=\sigma_{1}\left(\nu_{3}\right)$ and $N_{1}=N_{1}\left(\nu_{3}, A, \sigma\right)$ such that, for any $0<A \leqslant A_{1}, \sigma \geqslant \sigma_{1}$ and $n \geqslant N_{1}$

$$
\begin{aligned}
& \sup _{x \in B_{n}}|x| \int_{E_{x}^{c}}|u| p_{n}^{*}(x, u) d u \leqslant C\left(\nu_{3}, A, \sigma\right), \\
& \sup _{x \in B_{n}}|x| \int_{E_{x}^{c}} u^{2} p_{n}^{*}(x, u) d u \leqslant C\left(\nu_{3}, A, \sigma\right) \sqrt{n}, \\
& \sup _{x \in B_{n}} x^{2} \int_{E_{x}} u^{2} \pi_{n}(x, u) d u \leqslant C\left(\nu_{3}, A, \sigma\right) \sqrt{n} .
\end{aligned}
$$

Having obtained $(1.12-1.16)$ we fix $A$ and $\sigma$ as $\sigma=\sigma_{1}\left(\nu_{3}\right), A=\min \left\{A_{0}\left(\nu_{3}\right), A_{1}\left(\nu_{3}, \sigma_{1}\left(\nu_{3}\right)\right)\right.$. Then the arguments mentioned above prove (1.6) for $n \geqslant \max \left\{N_{1}, N_{0}\right\}$ and hence the theorem.

The proof of $(1.12$ - 13) follows the lines of that of theorem of [6] and will be omitted in the present paper. In what follows we prove $(1.14-16)$. We shall suppress the dependence of constants on $\nu_{3}, A$ and $\sigma$ except for $A_{1}$ and $\sigma_{1}$. Only the dependence on some other parameters (e.g. $\lambda$ in Lemma 1) will be indicated.

Introduce the function

$$
q_{n}(x, u)=\frac{1+x^{2}}{R} p_{n}^{*}(x, u)
$$

where

$$
R=E\left(1+S_{n}^{* 2}\right)=1+E \tilde{X}_{1}^{2}+\frac{1}{n} E \eta^{2} \leqslant C
$$

Let

$$
q_{n}(u)=\sup _{x} q_{n}(x, u), \quad q_{n}^{(1)}(u)=\sup _{x}\left|D_{x} q_{n}(x, u)\right|
$$

and, for $z>0$,

$$
Q_{n}(z)=\int_{|v| \geqslant z} q_{n}(\nu) d v, \quad Q_{n}^{(1)}(z)=\int_{|v| \geqslant z} q_{n}^{(1)}(v) d v .
$$


The proof of $(1.14-16)$ is based on the following lemma the proof of which will be given in Section 2.

LEMma 1. There exist positive $A_{1}=A_{1}\left(\nu_{3}, \sigma\right), \sigma_{1}=\sigma_{1}\left(\nu_{3}\right)$ and integers $m=m(\lambda) \geqslant 3, N_{2}=N_{2}(\lambda)$ such that for all $n \geqslant N_{2}, A \leqslant A_{1}, \sigma \geqslant \sigma_{1}$

$$
\int q_{n}(v) d v \leqslant C, \quad \int q_{n}^{(1)}(v) d v \leqslant C
$$

and for all $n \geqslant N_{2}, A \leqslant A_{1}, \sigma \geqslant \sigma_{1}, z>0$ and $\lambda \geqslant 1$

$$
\begin{aligned}
& Q_{n}(z) \leqslant C \cdot n \cdot P\left\{\left|Y_{1}^{*}\right|>\frac{z \sqrt{n}}{\max (\lambda m, \sigma)}\right\}+C(\lambda) \frac{n^{\lambda / 4}}{z^{3 \lambda / 2}}, \\
& Q_{n}^{(1)}(z) \leqslant C \cdot \frac{n^{1 / 4}}{z^{3 / 2}} .
\end{aligned}
$$

Note that (1.22) implies

$$
Q_{n}(z) \leqslant C \cdot \frac{n^{1 / 4}}{z^{3 / 2}}
$$

Proof of (1.14). By (1.17) and (1.18) we have (see (1.11))

$$
\begin{aligned}
& |x| \int_{E_{n}^{c}}|u| p_{n}^{*}(x, u) d u \leqq \frac{R|x|}{1+x^{2}} \int_{E_{n}^{c}}|u| q_{n}(u) d u \leqq \\
& \leqq \frac{R|x|}{1+x^{2}} \int_{a_{n}(x)}^{\infty} Q_{n}(u) d u .
\end{aligned}
$$

(We have used here that $\lim _{z \rightarrow \infty} z Q_{n}(z)=0$ by (1.24).) The proof of (1.14) obtains now by a straightforward application of (1.24).

Proof of (1.15). Likewise, the integral in (1.15) is bounded by

$$
\frac{R}{1+x^{2}} \int_{a_{n}(x)}^{\infty} u Q_{n}(u) d u
$$

To obtain this we have yet to show that

$$
\lim _{u \rightarrow+\infty} u^{2} Q_{n}(u)=0
$$

In order to prove (1.26) and to estimate (1.25) we use (1.22) with $\lambda=2$.

Note that letting $b=-E Y_{i} 1_{\left\{\left|Y_{i}\right| \leqslant n-1\right\}}$ (see (1.8)) we have

$$
0<b=E\left(X_{i}^{2}-1\right) \cdot 1_{\left\{\left|X_{i}\right|>\sqrt{n}\right\}} \leqslant \frac{\nu_{3}}{\sqrt{n}}\left(\leqslant 1 \text { for } n \geqslant \nu_{3}^{2}\right) .
$$

Hence $\left|\tilde{Y}_{i}\right| \leqslant n$ for $n \geqslant \nu_{3}^{2}$. Moreover we have for $n \geqslant \nu_{3}^{2}$

$$
E\left|\tilde{Y}_{1}\right|^{3 / 2}=E\left|X_{1}^{2}-1+b\right|^{3 / 2} \cdot 1_{\left\{\left|X_{1}\right| \leqslant \sqrt{n}\right\}} \leqslant\left(\nu_{3}^{2 / 3}+1-b\right)^{3 / 2} \leqslant 2^{3 / 2} \nu_{3} .
$$

Now we obtain for the probability in (1.22) with $\lambda=2$

$$
\begin{aligned}
& P\left\{\left|Y_{1}^{*}\right|>\frac{z \sqrt{n}}{2 m}\right\} \leqslant P\left\{\left|\tilde{Y}_{1}\right|>\frac{z \sqrt{n}}{4 m}\right\}+P\left\{\left|\xi_{1}\right|>\frac{z \sqrt{n}}{4 m}\right\}, \\
& P\left\{\left|\tilde{Y}_{1}\right|>\frac{z \sqrt{n}}{4 m}\right\} \leqslant 2^{3 / 2} \nu_{3} \frac{(4 m)^{3 / 2}}{z^{3 / 2} n^{3 / 4}} \text { for } z>0, \\
& P\left\{\tilde{Y}_{1}>\frac{z \sqrt{n}}{4 m}\right\}=0 \text { for } z>4 m n^{1 / 2},
\end{aligned}
$$




$$
P\left\{|\xi|>\frac{z \sqrt{n}}{4 m}\right\} \leqslant \frac{(4 m)^{4}}{z^{4} n^{2}} 3 \sigma^{4} \text { for } z>0 .
$$

Making use of (1.22), (1.28 - 31) one directly verifies (1.26) and obtains a bound for (1.25) which proves (1.15).

Proof of (1.16). It follows from (1.17- 19) that

$$
\left|D_{y} p_{n}^{*}(y, u)\right| \leqslant C\left[\frac{q_{n}^{(1)}(u)}{1+y^{2}}+\frac{2|y|}{\left(1+y^{2}\right)^{2}} q_{n}(u)\right]
$$

Hence the left hand side of (1.16) is not greater than

$$
C \sup _{x \in B_{n}}\left[\sup _{y:|y-x| \leqslant 1} \frac{x^{2}}{1+y^{2}} \int_{|u| \leqslant a_{n}(x)} u^{2} q_{n}^{(1)}(u) d u+\sup _{y:|y-x| \leqslant 1} \frac{2 x^{2}|y|}{\left(1+y^{2}\right)^{2}} \int_{|u| \leqslant a_{n}(x)} u^{2} q_{n}(u) d u\right] .
$$

Note that $\sup _{y:|y-x| \leqslant 1} \frac{x^{2}}{1+y^{2}} \leqslant 2$ and $\sup _{y:|y-x| \leqslant 1} \frac{x^{2}|y|}{\left(1+y^{2}\right)^{2}} \leqslant 1$ for all $x \in R$. Now (1.16) is verified directly using (1.23) and (1.24). Thus the proof of Theorem is completed.

2. Proof of Lemma 1.

Note that after we prove (1.21) it is sufficient to prove (1.22) and (1.23) for $Z \geqslant n^{1 / 6}$. Denote by $f_{n}(s, t)$ and $f_{n}^{*}(s, t)$ the characteristic functions of $\left(\tilde{S}_{n}, T_{n}^{*}\right)$ and $\left(S_{n}^{*}, T_{n}^{*}\right)$ respectively. Let

$$
g_{n}(s, u)=\frac{1}{2 \pi} \int e^{-i t u} f_{n}(s, t) d t, \quad g_{n}^{*}(s, u)=\frac{1}{2 \pi} \int e^{-i t u} f_{n}^{*}(s, t) d t .
$$

Then

$$
p_{n}^{*}(x, u)=\frac{1}{2 \pi} \int e^{-i s x} g_{n}^{*}(s, u) d s .
$$

Hence we have (see (1.17))

$$
R q_{n}(x, u)=\frac{1}{2 \pi} \int e^{-i s x}\left[g_{n}^{*}(s, u)-D_{s}^{2} g_{n}^{*}(s, u)\right] d s
$$

whence noting that $R>1$ (see (1.18)) we obtain

$$
\begin{aligned}
& Q_{n}(z) \leqslant \frac{1}{2 \pi} \int_{|u|>z}\left|g_{n}^{*}(s, u)-D_{s}^{2} g_{n}^{*}(s, u)\right| d u d s, \\
& Q_{n}^{(1)}(z) \leqslant \frac{1}{2 \pi} \int_{|u|>z}|s|\left|g_{n}^{*}(s, u)-D_{s}^{2} g_{n}^{*}(s, u)\right| d u d s .
\end{aligned}
$$

Let $\theta_{n}(s)=\theta\left(\frac{s}{A \sqrt{n}}\right)$, then (1.9) implies that

$$
g_{n}^{*}(s, u)=\theta_{n}(s) \cdot g_{n}(s, u) \text { and } g_{n}^{*}(s, u)=0 \text { for }|s| \geqslant A \sqrt{n} \text {. }
$$

Thus (2.4) and (2.5) are rewritten as

$$
\begin{aligned}
& Q_{n}^{(j)}(z) \leqslant \frac{1}{2 \pi} \int_{|s| \leqslant A} \int_{n}|s|>z||^{j}\left[\left|\theta_{n}(s)-D_{s}^{2} \theta_{n}(s)\right| \cdot\left|g_{n}(s, u)\right|+2\left|D_{s} \theta_{n}(s)\right| \cdot\right. \\
& \left.\left|D_{s} g_{n}(s, u)\right|+\left|\theta_{n}(s)\right|\left|D_{s}^{2} g_{n}(s, u)\right|\right] d s d u, j=0,1,
\end{aligned}
$$

with $Q_{n}^{(0)}=Q_{n}$. We shall prove that there exist $A_{1}\left(\nu_{3}, \sigma\right), \sigma_{1}\left(\nu_{3}\right)$, and integers $m>0, N_{2}>0$ such that for $n \geqq N_{2}, A \leqq A_{1}, \sigma \geqq \sigma_{1}$ and for $|s| \leqq A_{1} \sqrt{n}$

$$
\int\left|D_{s}^{i} g_{n}(s, u)\right| d u \leqslant C e^{-c s^{2}}(1+|s|)^{j}, j=0,1,2 \text {, }
$$

and for any $\lambda \geqslant 1, z \geqslant n^{1 / 6}$ 


$$
\begin{aligned}
& \int_{|u|>z}\left|D_{s}^{j} g_{n}(s, u)\right| d u \leqslant C\left.\leqslant, \nu_{3}\right) e^{-c s^{2}}(1+|s|)^{j} \\
& \cdot\left[n P\left\{Y_{1}^{*}>\frac{z \sqrt{n}}{\max \{m \lambda, 6\}}\right\}+n^{\lambda / 4} z^{-3 \lambda / 2}\right], \quad j=0,1,2
\end{aligned}
$$

where $D_{s}^{0} g=g$.

Then, by putting (2.7), (2.8), into (2.6), we obtain $(1.21-23)$. Proceeding to the proof of $(2.7)$, (2.8), introduce the functions

$$
\begin{aligned}
\Gamma_{k}(s, u)=E\left\{\exp \left[i s \sum_{i=1}^{k} \tilde{X}_{i}\right] \mid \sum_{i=1}^{k} Y_{i}^{*}\right. & =u\} p^{k^{*}}(u), \\
k & =1,2, \ldots
\end{aligned}
$$

where $p(u)$ denotes the density of $Y_{1}^{*}$ and the superscript $k^{*}$ denotes the $\mathrm{k}$-fold convolution. Note that (cf. (2.1))

$$
\sqrt{n} \Gamma_{n}\left(\frac{s}{\sqrt{n}}, u \sqrt{n}\right)=g_{n}(s, u)
$$

These functions have the following properties

$$
\begin{aligned}
& \left|\Gamma_{k}(s, u)\right| \leqslant p^{k^{*}}(u), \text { hence } \int\left|\Gamma_{k}(s, u)\right| d u \leqslant 1, \\
& \Gamma_{k}(s, \cdot)=\Gamma_{1}^{k^{*}}(s, \cdot)
\end{aligned}
$$

(see (3.9) in [7]).

LEMMA 2. There exists $\sigma_{0}=\sigma_{0}\left(\nu_{3}\right)$ such that for any $\sigma \geqslant \sigma_{0}$ there exist constants $C_{1}>0, C_{2}>0, A_{1}>0$, an integer $m>0$ and a function $B(u) \geqslant 0$ which depend on $\nu_{3}$ and $\sigma$ such that

$$
\left|\Gamma_{m}(t, u)\right| \leqslant p^{m^{*}}(u)-B(u) t^{2} \text { for }|t| \leqslant A_{1}
$$

and

$$
\int_{|u| \leqslant C_{2}} B(u) d u \geqslant C_{1}
$$

The proof will be given in Section 3.

COROLlaRY. For any $n \geqslant 2 m$

$$
\int\left|\Gamma_{n}(t, u)\right| d u \leqslant e^{-C_{1} t^{2} \frac{n}{2 m}} \text { for }|t| \leqslant A_{1}
$$

Proof. Write $n=a \cdot m+k$ with $a=[n / m], 0 \leqslant k<m$, Then, by (2.13)

$$
\begin{aligned}
& \int\left|\Gamma_{n}(t, u)\right| d u=\int \Gamma_{m}^{a^{*}}(t, \cdot)^{*} \Gamma_{k}(t, u) \mid d u \leqslant\left[\int\left|\Gamma_{m}(t, u)\right| d u\right]^{a} \leqslant \\
& \leqslant\left(1-C_{1} t^{2}\right)^{a} \leqslant \exp \left\{-C_{1} a t^{2}\right\} .
\end{aligned}
$$

It remains to note that $a \geqslant \frac{n}{2 m}$ for $n \geqslant 2 m$.

The constants $A_{1}$ and $\sigma_{1}$ which enter into the assertion concerning $(2.7-8)$ can now be specified as follows. We take $A_{1}$ as given by Lemma 2 and let $\sigma_{1}=\sigma_{0} \sqrt{2}$. The latter choice is needed to permit us the application of Lemma 2 and its corollary with $\sigma^{2} / 2$ instead of $\sigma^{2}$ (see (2.16)).

For the proof of (2.7) with $j=1,2$ we regard $\xi_{i}$ (see (1.8) and the paragraph before that) as a sum of two independent r.v.'s $\xi_{i}^{(1)}, \xi_{i}^{(2)}$ distributed $N\left(0, \frac{\sigma^{2}}{2}\right)$. Let $Y_{i}=\tilde{Y}_{i}+\xi_{i}^{(1)}$, then $Y_{i}^{*}=Y_{i}+\xi_{i}^{(2)}$. Let (cf. 
(2.9))

$$
\stackrel{\vee}{\Gamma}_{k}(s, u)=E\left\{\exp \left[i s \sum_{i=1}^{k} \tilde{X}_{i}\right] \sum_{i=1}^{k} \stackrel{v}{Y}_{i}=u\right\} \cdot{ }^{k^{*}}(u), \quad k=1,2, \ldots
$$

where $\stackrel{v}{p}(u)$ denotes the density of $\stackrel{\vee}{Y}_{i}$. Denote the density of $N\left(0, k \sigma^{2} / 2\right)$ by $\phi_{k}(u)$,

$$
\phi_{k}(u)=\frac{1}{\sqrt{\pi k \sigma}} e^{-u^{2} / k \sigma}, \quad k>0 .
$$

Then we have

$$
\Gamma_{k}(s, u)=\stackrel{\vee}{\Gamma}_{k}(s,)^{*} \phi_{k}(u), \quad k=1,2, \ldots
$$

Now, by (2.10), (2.12) and (2.18), we obtain that the left hand side of (2.7) with $j=1$ is

$$
\begin{aligned}
& \sqrt{n} \int \mid \stackrel{\vee}{\Gamma}_{n-1}\left(\frac{s}{\sqrt{n}}, \cdot\right)^{*} \stackrel{\vee}{\Gamma}{ }_{1}^{\prime}\left(\frac{s}{\sqrt{n}},\right)^{*} \phi_{n}(u)\left|d u \leqslant \sqrt{n} \int\right| \stackrel{\vee}{\Gamma^{\prime}}{ }_{1}\left(\frac{s}{\sqrt{n}},\right)^{*} \phi_{n}(u) \mid d u . \\
& \cdot \int\left|\stackrel{\vee}{\Gamma}_{n-1}\left(\frac{s}{\sqrt{n}}, u\right)\right| d u,
\end{aligned}
$$

where

$$
\stackrel{\vee}{\Gamma}_{1}^{\prime}(t, \cdot)=D_{t} \stackrel{\vee}{\Gamma}_{1}(t, \cdot) \text {. }
$$

Applying the corollary (see (2.15)) we get a bound $e^{-c s^{2}}$ for the last integral which holds for $|s| \leqslant A_{1}$. Thus we have to show that

$$
\sqrt{n} \int\left|{ }^{\vee}{ }^{\prime}\left(\frac{s}{\sqrt{n}}, \cdot\right)^{*} \phi_{n}(u)\right| d u \leqslant C(1+|s|) .
$$

Let

$$
\mu(u)=E\left\{\tilde{X}_{1} \mid \check{Y}_{1}=u\right\} \stackrel{\vee}{p}(u)
$$

Then

$$
\stackrel{\vee}{\Gamma}_{1}^{\prime}(t, u)=i \mu(u)+i E\left\{\tilde{X}_{1}\left[\exp \left(i t \tilde{X}_{1}\right)-1\right] \mid \stackrel{\vee}{Y_{1}}=u\right\} p(u) .
$$

We have

$$
\int \mu(u) d u=E \tilde{X}_{1}=0
$$

Let

$$
\dot{\circ}(u)=\int_{-\infty}^{u} \mu(v) d v \quad\left(=-\int_{u}^{\infty} \mu(v) d v \quad \text { by }(2.22)\right)
$$

Then

$$
\begin{aligned}
& \int|\mu(u)| d u \leqslant \int_{-\infty}^{0} \int_{-\infty}^{u}|\mu(v)| d v d u+\int_{0}^{\infty} \int_{u}^{\infty}|\mu(v)| d v d u= \\
& =\int|u \mu(u)| d u \leqslant E\left|\tilde{X}_{1} \stackrel{Y}{Y}_{1}\right| \leqslant C .
\end{aligned}
$$

Consider now the two terms arising when we put (2.21) into (2.20). The first of them is

$$
\sqrt{n} \int\left|\mu^{*} \phi_{n}(u)\right| d u=\sqrt{n} \int\left|\mu^{*} \phi_{n}^{\prime}(u)\right| d u \leqslant
$$




$$
\leqslant \int|\mu(u)| d u \cdot \sqrt{n} \int\left|\phi_{n}^{\prime}(u)\right| d u \leqslant C
$$

(see (2.23) and (2.17)). The second one (with $t=\frac{s}{\sqrt{n}}$ ) is not greater than $|s| E \tilde{X}_{1}^{2} \leqslant C|s|$, which together with (2.24) proves (2.20), and hence (2.7) with $j=1$.

In the way similar to (2.19) we obtain that the left hand side of (2.7) with $j=2$ is not greater than

$$
\begin{aligned}
& (n-1) \int\left|\Gamma_{1}^{\prime}\left(\frac{s}{\sqrt{n}}, \cdot\right)\right|^{2^{*} *} \Gamma_{n-2}\left(\frac{s}{\sqrt{n}}, \cdot\right)^{*} \phi_{n}(u)\left|d u+\int\right| \Gamma_{1}^{\prime \prime}\left(\frac{s}{\sqrt{n}}, \cdot\right)^{*} \Gamma_{n-2}\left(\frac{s}{\sqrt{n}}, \cdot\right)^{*} \\
& { }^{*} \phi_{n}(u)\left|d u \leqslant\left(\sqrt{n} \int\left|\stackrel{\Gamma}{\Gamma}_{1}^{\prime}\left(\frac{s}{\sqrt{n}}, \cdot\right)^{*} \phi \frac{n}{2}(u)\right| d u\right)^{2} \int\right| \check{\Gamma}_{n-1}\left(\frac{s}{\sqrt{n}}, u\right) \mid d u+ \\
& +\int \stackrel{\vee}{\Gamma_{1}}{ }^{\prime \prime}\left(\frac{s}{\sqrt{n}}, u\right)\left|d u \int \stackrel{\vee}{\Gamma}_{n-2}\left(\frac{s}{\sqrt{n}}, u\right)\right| d u \int \phi_{n}(u) d u .
\end{aligned}
$$

Now (2.7) with $j=2$ follows from (2.11),

$$
\int\left|\Gamma_{1}^{\prime \prime}(t, u)\right| d u \leqslant E\left|\tilde{X}_{1}\right|^{2}
$$

and an analoque of (2.20) with $\phi_{n}$ replaced by $\phi_{n / 2}$.

Turn to the proof of (2.8). We shall use some arguments from the proof of Theorem 1.2 in [7]. Let

$$
\rho_{m}(s, u)=p^{m^{*}}(u)-B(u) s^{2}, \quad \rho_{k}(u)=p^{k^{*}}(u), \quad k=1,2, \ldots
$$

and for $y>0$,

$$
\rho_{m}(s, u, y)=\rho_{m}(s, u) \cdot 1_{\{u<y\}}, \quad \rho_{k}(u, y)=\rho_{k}(u) \cdot 1_{\{u \leqslant y\}} .
$$

By Lemma 2 and (2.10), we have

$$
\int_{|u| \geqslant z}\left|g_{n}(s, u)\right| d u=\int_{|u|>z \sqrt{n}}\left|\Gamma_{n}\left(\frac{s}{\sqrt{n}}, u\right)\right| d u \leqslant \int_{|u|>z \sqrt{n}} \rho_{m}^{a^{*}}\left(\frac{s}{\sqrt{n}}, u\right)^{*} \rho_{k}(u) d u
$$

where $a$ and $k$ are as in the proof of the corollary to Lemma 2.

Hence

$$
\int_{|u|>z}\left|g_{n}(s, u)\right| d u \leqslant I_{1}(s, z)+I_{2}(s, z),
$$

where

$$
\begin{aligned}
& I_{1}(s, z)=\int_{|u|>z \sqrt{n}}\left[\rho_{m}^{a^{*}}\left(\frac{s}{\sqrt{n}}, \cdot\right)^{*} \rho_{k}(u)-\rho_{m}^{a^{*}}\left(\frac{s}{\sqrt{n}}, ; y\right)^{*} \rho_{k}(u, y)\right] d u, \\
& I_{2}(s, z)=\int_{|u|>z \sqrt{n}} \rho_{m}^{a^{*}}\left(\frac{s}{\sqrt{n}}, ; y\right)^{*} \rho_{k}(u, y) d u .
\end{aligned}
$$

The integrand in (2.31) is non-negative, hence we may extend the domain of integration to the whole real line. Thus we obtain

$$
\begin{aligned}
& I_{1}(s, z) \leqslant\left(\int \rho_{m}\left(\frac{s}{\sqrt{n}}, u\right) d u\right)^{a}-\left(\int \rho_{m}\left(\frac{s}{\sqrt{n}}, u, y\right) d u\right)^{a} \int \rho_{k}(u, y) d u \leqslant \\
& \leqslant a\left(1-\frac{C_{1}}{n} s^{2}\right)^{a-1} P\left\{\sum_{i=1}^{m} Y_{i}^{*}>y\right\}+\left(1-\frac{C_{1}}{n} s^{2}\right)^{a} P\left\{\sum_{i=1}^{k} Y_{i}^{*}>y\right\} .
\end{aligned}
$$

Note that $a-1 \geqslant n / 2 m$ for $n \geqslant 4 m$ and

$$
P\left\{\sum_{i=1}^{m} Y_{i}^{*}>y\right\} \leqslant m P\left\{Y_{1}^{*}>\frac{y}{m}\right\} \text {. }
$$


Therefore

$$
\begin{aligned}
& I_{1}(s, z) \leqslant e^{-\left(C_{1} / 2 m\right) s^{2}}\left[a \cdot m \cdot P\left\{Y_{1}^{*}>\frac{y}{m}\right\}+k P\left\{Y_{1}^{*}>\frac{y}{k}\right\}\right] \leqslant \\
& \leqslant e^{-\left(C_{1} / 2 m\right) s^{2}} n \cdot P\left\{Y_{1}^{*}>\frac{y}{m}\right\} .
\end{aligned}
$$

Put $y=\frac{z \sqrt{n}}{\lambda}$. Then (2.33) gives a part of the right hand side of (2.8).

To estimate $I_{2}(s, z)$ we apply the arguments from [7] (see (3.25), (3.32) - (3.33) of [7]) to obtain that for an arbitrary $h>0$

$$
I_{2}(s, z) \leqslant e^{-h z \sqrt{n}}\left[\int e^{h u} \rho_{m}\left(\frac{s}{\sqrt{u}}, u, y\right) d u\right]^{a} R_{k}(h, y)
$$

with

$$
R_{k}(h, y)=\int_{-\infty}^{y} e^{h u} p^{k^{*}}(u) d u \leqslant 1+2 k \nu_{3} / 2 e^{h y} y^{-3 / 2}
$$

where

$$
\nu_{3 / 2}=E\left|Y_{1}^{*}\right|^{3 / 2} \leqslant C
$$

By (2.27), (2.28) we have

$$
\int e^{h u} \rho_{m}\left(\frac{s}{\sqrt{n}}, u, y\right) d u \leqslant R_{m}(h, y)-\frac{s^{2}}{n} \int_{-\infty}^{y} e^{h u} B(u) d u .
$$

Put

$$
y=\frac{z \sqrt{n}}{\lambda}, \quad h=\frac{1}{y} \log \left(y^{3 / 2} / n\right)
$$

Then

$$
e^{-h z \sqrt{n}}=n^{\lambda / 4} \lambda^{3 \lambda / 2} z^{-3 \lambda / 2}
$$

and, by (2.35)

$$
R_{k}(h, y) \leqslant 1+2 k \nu_{3 / 2} n^{-1}, \quad R_{m}(h, y) \leqslant 1+2 m \nu_{3 / 2} n^{-1} .
$$

Further, since $z \geqslant n^{1 / 6}$ we have $y \geqslant \frac{n^{2 / 3}}{\lambda}$ and $y \geqslant C_{2}$ (see $(2.14)$ ) for $n \geqslant\left(\lambda C_{2}\right)^{3 / 2}$. Moreover, $h \leqslant \frac{3}{2} e^{-1} n^{-2 / 3} \leqslant \frac{1}{2}$ for $n \geqslant 2$. Therefore, by (2.14)

$$
\int_{-\infty}^{y} e^{h u} B(u) d u \geqslant e^{-h C_{2}} C_{1} \geqslant C_{3}=C_{1} e^{-C_{2} / 2}
$$

Putting (2.37), (2.38) into (2.36) and then into (2.34) we obtain

$$
I_{2}(s, z) \leqslant \lambda^{3 \lambda / 2} n^{\lambda / 4} z^{-3 \lambda / 2} \exp \left(2 \nu_{3 / 2}-C_{3} \frac{a}{n} s^{2}\right)
$$

Note that $\frac{a}{n} \geqslant \frac{1}{2 m}$ for $n \geqslant 2 m$. Now (2.33) and (2.39) imply (2.8) with $j=0$.

In a way similar to (2.20) we see that the left-hand side of (2.8) with $j=1$ is

$$
\sqrt{n} \int_{|u|>z \sqrt{n}}\left|\stackrel{\vee}{\Gamma}_{n-1}\left(\frac{s}{\sqrt{n}}, \cdot\right)^{*} \Gamma_{1}^{\prime}\left(\frac{s}{\sqrt{n}}, \cdot\right)^{*} \phi_{n}(u)\right| d u=J_{1}+J_{2}
$$


where $J_{1}$ and $J_{2}$ are the integrals over $u>z \sqrt{n}$ and $u<-z \sqrt{n}$ respectively.

We shall use the following inequality: for two non-negative functions, $f$ and $g$,

$$
\int_{u>y} f^{*} g(u) d u=\int_{u+v>y} \int f(u) g(v) d u d v \leqslant \int_{u>\frac{y}{2}} f(u) d u \int g(v) d v+\int f(u) d u \int_{v>\frac{y}{2}} g(v) d v .
$$

Thus

$$
\begin{aligned}
& J_{1} \leqslant \sqrt{n} \int_{u>z \sqrt{n} / 2}\left|\check{\Gamma}_{1}^{\prime}\left(\frac{s}{\sqrt{n}}, \cdot\right) \phi_{n}(u)\right| d u \cdot \int\left|\stackrel{\vee}{\Gamma}_{n-1}\left(\frac{s}{\sqrt{n}}, u\right)\right| d u+ \\
& \sqrt{n} \int\left|\stackrel{\Gamma}{\Gamma}_{1}^{\prime}\left(\frac{s}{\sqrt{n}}, \cdot\right) \phi_{n}(u)\right| d u \cdot \int_{u>z \sqrt{n} / 2}\left|\check{\Gamma}_{n-1}\left(\frac{s}{\sqrt{n}}, u\right)\right| d u .
\end{aligned}
$$

Note that

$$
\left|E X_{1} \cdot 1_{\left\{\left|X_{1}\right| \leqslant \sqrt{n}\right\}}\right| \leqslant E\left|X_{1}\right| \leqslant\left(E X_{1}^{2}\right)^{1 / 2}=1 .
$$

Hence $\left|\tilde{X}_{1}\right| \leqslant \sqrt{n}+1 \leqslant 2 \sqrt{n}$ (see (1.7)). Therefore

$$
\left|\stackrel{\vee}{\Gamma}_{1}^{\prime}(t, u)\right|=\mid E\left[\tilde{X}_{1} e^{i t X_{1}} \mid \breve{Y}_{1}=u \underline{v}(u) \leqslant 2 \sqrt{n} p(u) .\right.
$$

Then noting that ${ }^{*} \phi_{1}(u)=p^{*}(u)$, we obtain

$$
\begin{aligned}
& \sqrt{n} \int_{u>z \sqrt{n} / 2}\left|\Gamma_{1}^{\prime}\left(\frac{s}{\sqrt{n}},\right)^{*} \phi_{n}(u)\right| d u \leqslant 2 n \int_{u>z \sqrt{n} / 2} p^{* *} \phi_{n-1}(u) d u \leqslant \\
& \leqslant 2 n P\left\{Y_{1}^{*}>\frac{z \sqrt{n}}{4}\right\}+2 n \int_{u>z \sqrt{n} / 4} \phi_{n-1}(u) d u .
\end{aligned}
$$

We have for the last term

$$
2 n \int_{u>z \sqrt{n} / 4} \phi_{n-1}(u) d u \leqslant 2 n \int_{u>z / 4} \phi_{1}(u) d u \leqslant \frac{C n}{z^{6}} \leqslant C n^{\lambda / 4} z^{-3 \lambda / 2}
$$

for $z \geqslant n^{1 / 6}$. Now we put (2.44) and (2.45) into (2.42). Moreover we use (2.20) and estimate the integrals of $\stackrel{\vee}{\Gamma}_{n-1}$ in the same way as those in (2.7) and (2.8) with $j=1$. The inequalities thus obtained together with similar inequalities for $J_{2}$ (see (2.40)) prove (2.8) with $j=1$.

In a similar way to (2.25) we obtain that the left hand side of (2.8) with $j=2$ is not greater than

$$
\begin{aligned}
& n \int_{|u|>z \sqrt{n}}\left|\left[\Gamma_{1}^{\prime}\left(\frac{s}{\sqrt{n}}, \cdot\right)\right]^{2^{*} *}{ }^{\vee} \Gamma_{n-2}\left(\frac{s}{\sqrt{n}}, \cdot\right)^{*} \phi_{n}(u)\right| d u+ \\
& +\int_{|u|<z \sqrt{n}}\left|{ }^{\prime}{ }_{1}^{\prime \prime}\left(\frac{s}{\sqrt{n}}, \cdot\right)^{*} \stackrel{\vee}{\Gamma}_{n-2}\left(\frac{s}{\sqrt{n}},\right)^{*} \phi_{n}(u)\right| d u=J_{1}+\ldots+J_{4}
\end{aligned}
$$

where the $J$ s are the parts of the integrals corresponding to integration over $u>z \sqrt{n}$ and $u<-z \sqrt{n}$ respectively.

We have

$$
\begin{aligned}
& J_{1} \leqslant 2 \sqrt{n} \int_{u>z \sqrt{n} / 3}\left|\Gamma_{1}^{\prime}\left(\frac{s}{\sqrt{n}}, \cdot\right)^{*} \phi \frac{n}{2}(u) d u \cdot \sqrt{n} \int\right| \Gamma_{1}^{\prime}\left(\frac{s}{\sqrt{n}}, \cdot\right)^{*} \phi \frac{n}{2}(u) \mid d u \\
& \cdot \int\left|\check{\Gamma}_{n-2}\left(\frac{s}{\sqrt{n}}, u\right)\right| d u+\left(\sqrt{n} \int\left|\breve{\Gamma}_{1}^{\prime}\left(\frac{s}{\sqrt{n}}, \cdot\right)^{*} \phi \frac{n}{2}(u)\right| d u\right)^{2} \cdot \\
& \cdot \int_{u>2 \sqrt{n} / 3}\left|\check{\Gamma}_{n-2}\left(\frac{s}{\sqrt{n}}, u\right)\right| d u,
\end{aligned}
$$




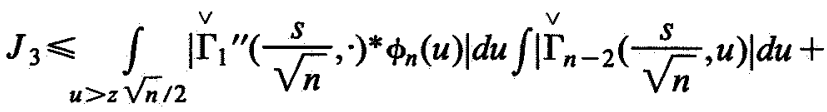

$$
\begin{aligned}
& +\int\left|\Gamma_{1}{ }^{\prime \prime}\left(\frac{s}{\sqrt{n}},\right)^{*} \phi_{n}(u)\right| d u \int_{u>2 \sqrt{n} / 2}\left|\stackrel{\vee}{\Gamma_{n-2}}\left(\frac{s}{\sqrt{n}}, u\right)\right| d u
\end{aligned}
$$

and similar relations for $J_{2}, J_{4}$ with corresponding change of the domains of integration.

In the first integral in (2.47) we have $\left|\Gamma_{1}{ }^{\prime \prime}(t, u)\right| \leqslant 4 n p(u)$ (cf. (2.43)). Then this integral is estimated as in (2.44), (2.45). The other integrals in (2.46), (2.47) are estimated in a similar way to (2.20), (2.44 - 45), (2.26) and (2.7), (2.8) with $j=0$. Thus the proof of $(2.7-8)$ and hence the lemma is completed.

\section{Proof of Lemia 2.}

Let $B \subset R^{2}$ be defined as

$$
B=\{(x, u):|x| \leqslant a,|u| \leqslant b\}, \quad a=8 v_{3}, \quad b=96 v_{3}^{2} .
$$

Denote by $\tilde{P}(d x, d y)$ the distribution of $\tilde{X}_{1}, \tilde{Y}_{1}$ (see $(1.7),(1.8)$ ) and let $P_{B}$ be the corresponding distribution conditional on $B$, i.e. for a Borel set $A \subset R^{2}$

$$
P_{B}(A)=\frac{\tilde{P}(A \cap B)}{\alpha}, \text { with } \alpha=\tilde{P}(B) .
$$

Consider i.i.d. random vectors $\left(Z_{0 i}, Z_{i}\right)$ distributed according to $P_{B}$. Denote by $\gamma_{0}$ and $\gamma$ the expectations and by $\sigma_{0 B}^{2}, \sigma_{B}^{2}$ the variances of $Z_{01}$ and $Z_{1}$ respectively. Moreover, let, as before, $\xi_{i}$ be i.i.d. and independent of $\left\{Z_{0 i}, Z_{i}\right\}$ normal $N\left(0, \sigma^{2}\right)$ r.v.'s and $Z_{i}^{*}=Z_{i}+\xi_{i}$. Let

$$
S_{n B}=n^{-1 / 2} \sum\left(Z_{0 i}-\gamma_{0}\right), \quad T_{n B}^{*}=n^{-1 / 2} \Sigma\left(Z_{i}^{*}-\gamma\right), \sigma_{B}^{* 2}=\operatorname{var}\left(Z_{i}^{*}\right) \text {. }
$$

Denote by $p_{B}(u)$ the density of $Z_{1}^{*}$.

First we shall show that $\alpha$ and $\sigma_{0 B}$ are bounded away from 0 . In a similar way to (1.27) we find that, for $n \geqslant 4 \nu_{3}^{2}(\geqslant 4)$,

$$
E\left|\tilde{X}_{1}\right| \leqslant 2 \nu_{3}, \quad E\left|\tilde{X}_{1} \tilde{Y}_{1}\right| \leqslant 3 \nu_{3}
$$

Then

$$
\begin{aligned}
& \tilde{P}\left(B^{c}\right) \leqslant \nu_{3}\left(\frac{2}{a^{3}}+\frac{2^{3 / 2}}{b^{3 / 2}}\right), \\
& \left|\int_{B} x d \tilde{P}\right|=\left|\int_{B^{c}} x d \tilde{P}\right| \leqslant \nu_{3}\left(\frac{2}{a^{2}}+\frac{3}{b}\right), \\
& \int_{B^{c}} x^{2} d \tilde{P} \leqslant \nu_{3}\left(\frac{2}{a}+\frac{3 a}{b}\right),
\end{aligned}
$$

and with $a$ and $b$ as given in (3.1),

$$
\begin{aligned}
\alpha & \geqslant \frac{127}{128}, \\
\sigma_{0 B}^{2} & \geqslant 1-\frac{1}{\alpha} \nu_{3}\left(\frac{2}{a}+\frac{3 a}{b}\right)-\frac{1}{\alpha^{2}} \nu_{3}^{2}\left(\frac{2}{a^{2}}+\frac{3}{b}\right)^{2} \geqslant \\
& \geqslant 1-\frac{1}{2}\left(\frac{128}{127}\right)^{2}>\frac{1}{3} .
\end{aligned}
$$

Note that $\sigma_{B}^{* 2}=\sigma_{B}^{2}+\sigma^{2}$ and $\operatorname{cov}\left(Z_{01}, Z_{1}^{*}\right)=\operatorname{cov}\left(Z_{01}, Z_{1}\right)$. Hence the correlation, $\rho$, between $Z_{01}$ and $Z_{1}^{*}$ satisfies the inequality

$$
|\rho| \leqslant \frac{\sigma_{B}}{\sqrt{\sigma_{B}^{2}+\sigma^{2}}} .
$$


Since $\sigma_{B} \leqslant b=96 \nu_{3}^{2}$ we have

$$
|\rho| \leqslant \frac{1}{\sqrt{2}} \text { whenever } \sigma \geqslant \sigma_{0}=96 \nu_{3}^{2}
$$

Let, further

$$
\Gamma_{k B}(s, u)=E\left\{\exp \left(i s \sum_{i=1}^{k} Z_{0 i}\right) \sum_{i=1}^{k} Z_{i}^{*}=u\right\} p_{B}^{k^{*}}(u), \quad k=1,2, \ldots
$$

Moreover, for $B^{c}=R^{2} \backslash B$ define $P_{B^{c}}$ and $\Gamma_{k B^{c}}$ in a similar way. Note that

$$
\alpha p_{B}(u)+(1-\alpha) p_{B^{c}}(u)=p^{*}(u),
$$

$p^{*}$ being the density of $Y_{1}^{*}=\tilde{Y}_{1}+\xi_{1}$ (see (1.8)),

$$
\alpha \Gamma_{1 B}(s, u)+(1-\alpha) \Gamma_{1 B^{c}}(s, u)=\Gamma_{1}(s, u),
$$

(see (2.9)) and

$$
\left|\Gamma_{k B}(s, u)\right| \leqslant p_{B}^{k^{*}}(u), \quad\left|\Gamma_{k B^{c}}(s, u)\right| \leqslant p_{B^{c}}^{k^{*}}(u) .
$$

LEMMA 3. For any $\sigma \geqslant \sigma_{0}=96 \nu_{3}^{2}$ there exist $A_{1}>0$, an integer $m>0$ and $d>0$ depending on $\nu_{3}$ and $\sigma$ such that, for $|s| \leqslant A_{1}$,

$$
\left|\Gamma_{m B}(s, u)\right| \leqslant p_{B}^{m^{*}}(u)-\sqrt{m} d s^{2} 1_{E}\left(\frac{u-m \gamma}{\sqrt{m}}\right),
$$

where $E=\left\{u:|u| \leqslant u_{0}\right\}$ with $u_{0}=\sigma_{0} \sqrt{2 \ln 2}$.

We shall use this lemma to finish the proof of Lemma 2 and after that its proof will be given. We have by (2.12) and (3.11) with $m$ as in Lemma 3

$$
\Gamma_{m}(s, \cdot)=\Gamma_{1}^{m^{*}}(s, \cdot)=\sum_{k=1}^{m} C_{m}^{k} \alpha^{k}(1-\alpha)^{m-k} \Gamma_{1 B}^{k^{*}}(s, \cdot)^{*} \Gamma_{1 B^{c}}^{m-k)^{*}}(s, \cdot)
$$

Now we estimate the last term by (3.13) and the other terms by (3.12). Then we employ (3.10) to obtain that for $|s| \leqslant A_{1}$

$$
\left|\Gamma_{m}(s, u)\right| \leqslant p^{m^{*}}(u)-s^{2} B(u),
$$

where

$$
B(u)=\alpha^{m} \sqrt{m} d 1_{E}\left(\frac{u-m \gamma}{\sqrt{m}}\right) .
$$

The length of the interval

$$
I=\left\{u: \frac{u-m \gamma}{\sqrt{m}} \in E\right\}
$$

is $2 \sqrt{m} u_{0}$ and $I \subset\left[-C_{2}, C_{2}\right]$ for $C_{2}=m a+u_{0} \sqrt{m}$ with $a$ defined in (3.1). Hence

$$
\int_{|u| \leqslant C_{2}} B(u) d u=C_{1}=2 u_{0} m \alpha^{m} d
$$

which completes the proof. 
4. Proof of Lemma 3.

Let $p_{k B}$ be the density of $T_{k B}^{*}$ (see (3.3)) and

$$
g_{k B}(s, u)=E\left\{\exp \left(i s S_{k B}\right) \mid T_{k B}^{*}=u\right\} p_{k B}(u), \quad k=1,2, \ldots
$$

Comparing (4.1) with (3.9) we have

$$
\Gamma_{k B}(s, u)=\frac{1}{\sqrt{k}} g_{k B}\left(s \sqrt{k}, \frac{u-k \gamma}{\sqrt{k}}\right), \quad k=1,2 \ldots
$$

Then (3.13) is equivalent to

$$
\left|g_{m B}(s, u)\right| \leqslant P_{m B}(u)-d s^{2} 1_{E}(u)
$$

for

$$
|s| \leqslant A_{2}=A_{1} \sqrt{m} \text {. }
$$

Let

$$
\mu_{j, k}(u)=E\left\{S_{k B}^{j} \mid T_{k B}^{*}=u\right\} p_{k B}(u), \quad j, k=1,2 \ldots
$$

Denote by $\phi_{B}(x, u)$ the density of the bivariate normal distribution with zero mean and the same covariance matrix as $\left(Z_{01}, Z_{1}^{*}\right)$. Recall that the correlation coefficient of this distribution satisfies (3.8). Let $\phi_{B}(u)$ be the marginal density,

$$
\begin{aligned}
& \phi_{B}(u)=\int \phi_{B}(x, u) d x, \text { and } \\
& \mu_{j}(u)=\int x^{j} \phi_{B}(x, u) d x, \quad j=1,2, \ldots
\end{aligned}
$$

LEMMA 4. There exist constants $L_{j}=L_{j}\left(\nu_{3}, \sigma\right), j=0,1, \ldots$ such that

$$
\begin{aligned}
& \sup _{u}\left|P_{k B}(u)-\phi_{B}(u)\right| \leqslant \frac{L_{0}}{\sqrt{k}}, \quad k=1,2, \ldots \\
& \sup _{u}\left|\mu_{j, k}(u)-\mu_{j}(u)\right| \leqslant \frac{L_{j}}{\sqrt{k}}, \quad k=1,2, \ldots
\end{aligned}
$$

The proof will be omitted. It uses some technique from [6] and is simplified by the fact that $Z_{0,1}, Z_{1}$ are bounded and $Z_{1}^{*}$ contains a normal component.

Expanding the exponential function in (4.1) by the Taylor formula we obtain

$$
g_{k B}(s, u)=\bar{g}_{k B}(s, u)+|s|^{3} \zeta_{k}(s, u)
$$

with

$$
\begin{aligned}
\bar{g}_{k B}(s, u) & =p_{k B}(u)+i s \mu_{1, k}(u)-\frac{s^{2}}{2} \mu_{2, k}(u) \\
\left|\zeta_{k}(s, u)\right| & \leqslant \frac{1}{6} E\left[\left|S_{k B}\right|^{3}\left|T_{k B}^{*}=u\right| p_{k B}(u) \leqslant\right. \\
& \leqslant \zeta_{k}(u)=\frac{1}{6}\left[p_{k B}(u)+\mu_{4, k}(u)\right] .
\end{aligned}
$$

We have

$$
\left|\bar{g}_{k B}(s, u)\right|^{2}=p_{k B}^{2}(u)\left[1-s^{2}\left(\frac{D_{k}(u)}{p_{k B}^{2}(u)}-\frac{s^{2}}{4} \frac{\mu_{2 k}^{2}(u)}{p_{k B}^{2}(u)}\right)\right],
$$

where

$$
D_{k}(u)=p_{k B}(u) \mu_{2 k}(u)-\mu_{1, k}^{2}(u) .
$$


It follows from (4.8) and (4.11) that

$$
\left|g_{k B}(s, u)\right| \leqslant p_{k B}(u)-\frac{s^{2}}{2}\left[\frac{D_{k}(u)}{p_{k B}(u)}-|s| \zeta_{k}(u)-\frac{s^{2}}{4} \frac{\mu_{2 k}^{2}(u)}{p_{k B}(u)}\right]
$$

Our aim is to find $m$ and $A_{2}$ such that the expression in brackets is bounded from below by a positive constant for $k=m,|s| \leqslant A_{2},|u| \leqslant u_{0}$. Since we consider $\sigma \geqslant \sigma_{0}$ and $\sigma_{B}^{*} \geqslant \sigma$, we have

$$
\phi_{B}(u) \geqslant \frac{1}{2} \phi_{B}(0) \text { for }|u| \leqslant u_{0}
$$

Moreover, since $\sigma_{B}^{*} \leqslant \sigma \sqrt{2}$, we have

$$
\phi_{B}(0)=\frac{1}{\sqrt{2 \pi} \sigma_{B}^{*}} \geqslant \frac{1}{2 \sigma \sqrt{\pi}} .
$$

Let $m_{1}$ be the smallest integer satisfying

$$
\frac{L_{0}}{\sqrt{m_{1}}} \leqslant \frac{1}{8 \sqrt{\pi} \sigma}\left(\leqslant \frac{1}{4} \phi_{B}(0)\right)
$$

(see (4.6)). Then (4.16) and (4.14) imply that for $|u| \leqslant u_{0}, k \geqslant m_{1}$

$$
p_{k B}(u) \geqslant \frac{1}{4} \phi_{B}(0), \quad \frac{\phi_{B}(u)}{p_{k B}(u)} \geqslant \frac{2}{3} \text {. }
$$

Let $D(u)=\phi_{B}(u) \mu_{2}(u)-\mu_{1}^{2}(u)$. Note that $D(u) / \phi_{B}^{2}(u)$ is the conditional variance of the first variable given the second one, i.e.

$$
D(u)=\sigma_{0 B}^{2}\left(1-\rho^{2}\right) \phi_{B}^{2}(u) \text {. }
$$

Write the first term in brackets in (4.13) as

$$
\frac{D_{k}(u)}{p_{k B}(u)}=\frac{D(u)}{p_{k B}(u)}+\frac{D_{k}(u)-D(u)}{p_{k B}(u)} .
$$

By (4.14), (4.15), (4.17) and (4.18) we have for $|u| \leqslant u_{0}, k \leqslant m_{1}$

$$
\frac{D(u)}{p_{k B}(u)}=\frac{D(u)}{\phi_{B}(u)} \cdot \frac{\phi_{B}(u)}{p_{k B}(u)} \geqslant \frac{1}{3} \sigma_{0 B}^{2}\left(1-\rho^{2}\right) \phi_{B}(0)
$$

whence, by (3.6), (3.8) and (4.15) we obtain

$$
\frac{D(u)}{p_{k B}(u)} \geqslant \frac{\phi_{B}(0)}{18} \geqslant \frac{1}{36 \sqrt{\pi} \sigma} \text { for }|u| \leqslant u_{0}, k \geqslant m_{1} .
$$

Further, we have

$$
\begin{aligned}
& D_{k}(u)-D(u)=\left[\mu_{2 k}(u)-\mu_{2}(u)\right] p_{k B}(u)+ \\
& {\left[p_{k B}(u)-\phi_{B}(u)\right] \mu_{2}(u)+\left[\mu_{1}(u)-\mu_{1 k}(u)\right]\left[\mu_{1}(u)+\mu_{1 k}(u)\right] .}
\end{aligned}
$$

It is seen from (4.5), Lemma 4 and the fact that $\sigma_{B}^{*} \geqslant \sigma_{0}$ that there exists a constant $L=L\left(\nu_{3}, \sigma\right)$ such that, for $\sigma \geqslant \sigma_{0}$

$$
\sup _{u}\left|D_{k}(u)-D(u)\right| \leqslant \frac{L}{\sqrt{k}}, \quad k=1,2, \ldots
$$

It follows from (4.17), (4.14) and (4.22) that we can find $m \geqslant m_{1}$ such that

$$
\frac{\left|D_{m}(u)-D(u)\right|}{p_{m B}(u)} \leqslant \frac{1}{72 \sqrt{\pi} \sigma} \text { for }|u| \leqslant u_{0}
$$


Let $d=\frac{1}{144 \sqrt{\pi} \sigma}$. Then (4.19), (4.21) and (4.23) imply that

$$
\frac{D_{m}(u)}{p_{m B}(u)} \geqslant 2 d \text { for }|u| \leqslant u_{0}
$$

By (4.17) and Lemma $4, \frac{\mu_{2 m}^{2}}{p_{m B}(u)}$ and $\zeta_{k}(u)$ are bounded for $|u| \leqslant u_{0}$, hence we can find $A_{2}>0$ such that

$$
\sup _{|u| \leqslant u_{0}}\left[\frac{A_{2}^{2}}{4} \frac{\mu_{2, k}^{2}(u)}{p_{m B}(u)}+A_{2} \zeta_{m}(u)\right] \leqslant d .
$$

Then (4.13), (4.24) and (4.25) imply (4.3).

\section{REFERENCES}

[1] Chung, K.L. (1946). The approximate distribution of Student's statistic. Ann. Math. Statist., $17,447-465$

[2] SCHAzFER, M. (1975). Das asymptotische Verhalten des $t$-Tests unter allgemeinen Alternativen. Math. Operationsforschung und Statist., 6, 775-785.

[3] Helmers, R. and VAN ZWET, W.R. (1982). The Berry-Esseen bound for $U$-statistics. Statistical Decision Theory and Related Topics III, Vol. 1, 497-512, S.S GUPTA and J.O. BerGER editors, Academic Press, New York

[4] Chibisov, D.M. (1982). Asymptotic expansions in problems of testing hypotheses. I, II. Izv. Akad. Nauk Uz SSR Ser.Fiz-Mat. Nauk, no 5, 18-26; no 6, 23-30 (Russian).

[5] Chrbisov, D.M (1980-81). Asymptotic expansion for the distribution of a statistic admitting a stochastic expansion. I, II. Teor. Verojatn. i Primenen., 25, 745-756, 26, 3-14 (Russian), Theory Prob. Appl., 25, 732-744, 26, 1-12.

[6] Chibisov, D.M. (1979). Asymptotic expansion for partial moments. Teor Verojatn. i Primenen., 24, 449-462 (Russian) Theory Prob. Appl., 24, 453-466.

[7] Chibisov, D.M (1980). Asymptotic behavior of partial densities and their derivatives. Teor. Verojatn. i Primenen., 25, 3-17 (Russian) Theory Prob. Appl., 25, 1-15. 
